\title{
MULTIPLE EPITHELIOMATA OF THE EYELIDS OF ONE EYE*
}

\author{
BY
}

\author{
A. LISTER AND V. M. DALLEY \\ London
}

THE following case of epithelioma of the conjunctiva developing after prolonged inflammation, first in the lower eyelid and then in the upper, is thought to be sufficiently unusual to be of interest.

\section{Case Report}

A man aged 67 first attended the London Hospital in April, 1954, with an inflamed left eye. It had been troubling him for 3 years. There was trichiasis due to scarring of the upper lid, which was thought, on somewhat doubtful grounds, to be caused by trachoma, grossly inflamed conjunctiva, and marginal corneal vascularization. Two initial cultures were negative but later Staphylococcus pyogenes was grown. The conjunctivitis continued unabated for the next 2 years in spite of treatment with albucid, silver nitrate, oxycyanide of mercury lotion, aureomycin, penicillin, saline lotion-when active treatment was thought to be aggravating rather than helping the condition-neomycin, systemic sulphonamides, cortisone, epilation, and meibomian massage.

In the Summer of 1956 the inner end of the left lower lid became markedly thickened (Fig. 1). A biopsy taken from it showed "poorly differentiated squamous cell carcinoma from the conjunctival surface of the lid". Radiotherapy, given at the Royal Marsden Hospital, was apparently successful in curing the growth, and there has been no recurrence since, although considerable conjunctival and corneal scarring and xerophthalmia also resulted. Enucleation was declined, and during the following year the eye became fairly quiet and comfortable.

In April, 1958, the inner end of the upper lid became swollen by what appeared to be a collection of pustules in the thickness of the tarsus. Biopsy showed a growth similar to that in the lower lid (Fig. 2). This, in its turn, has responded to radiotherapy, and the eye is now fairly quiet and comfortable, although there is considerable symblepharon and corneal scarring. The right eye remains healthy, with visual acuity $6 / 6$.

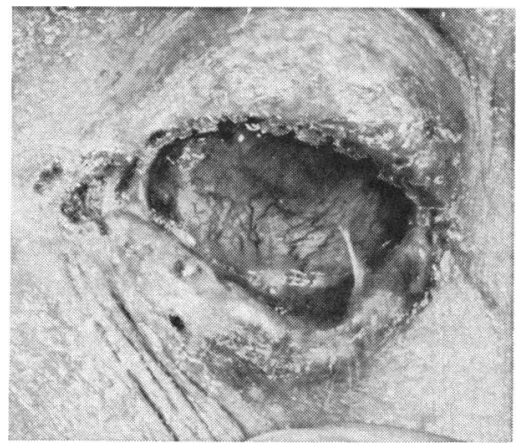

Fig. 1. Before starting first course of treatment to lower lid, July, 1956.

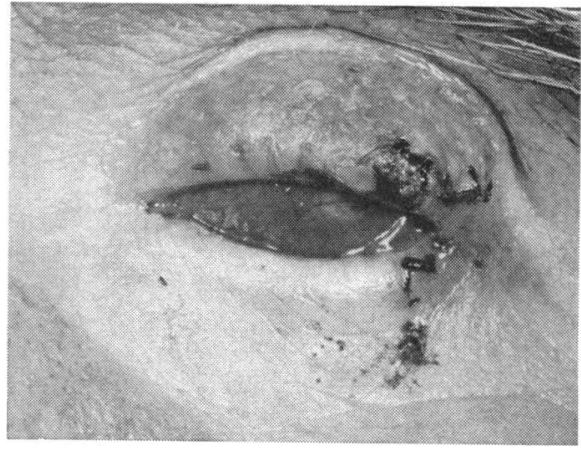

Fig. 2.-New lesion on upper lid with radiation changes in lower lid, May, 1958. 
Radiotherapy Record.-The patient was referred to the Royal Marsden Hospital in July, 1956, with a diagnosis of epithelioma of the conjunctival surface of the outer half of the left lower lid. In view of the state of the eye before the treatment and a long-standing history of severe seborrhoeic dermatitis of face and scalp, it was felt that external radiation rather than interstitial radon seeds should be used, since by this method the treatment and any ensuing reactions could be more easily controlled. $60 \mathrm{kV} x$ rays were used, a dose of $6,200 r$ being given in daily doses of $400 \mathrm{r}$. The anterior part of the eye was protected by a lead shield, which was inserted beneath the lid after local anaesthesia.

There was little effect on the eye or reaction of the eyelid during treatment. The part of the lid affected by dermatitis and included in the treatment area appeared to be improved by $x$ rays. A fortnight after the end of treatment, the lesion had healed, although the skin was still oedematous and some crusting was present. In spite of the care taken to protect the eye, an acute iritis supervened at this stage.

From this time until May, 1958, the lid remained healed but the eye was intensely painful and its removal was considered. At this time the left upper lid was noticed to be nodular in its outer part and a biopsy showed epithelioma. Almost the whole of the outer half of the lid was involved, the upper extent being the bony margin of the orbit.

Radiotherapy was used for the second treatment in view of the obviously unstable state of the skin, the problem being to protect both the anterior surface of the eye and also the previously treated area. The conjunctival sac was now unusually small owing to scarring and a normal-sized eye-shield could not be used. A special spoon-shaped shield was made (Fig. 3), and the lower lid was protected from further radiation throughout the treatment (Fig. 4). 60kV apparatus was used with a light device to facilitate the accurate use of the various protective measures and a dose of 5,600 r was given.

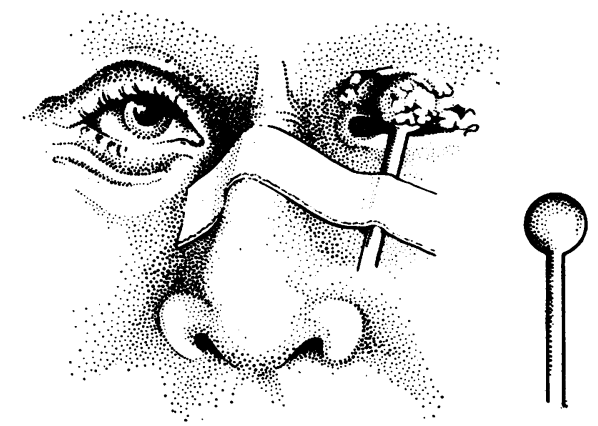

FIG. 3.-Diagram of corneal shield in place during treatment.

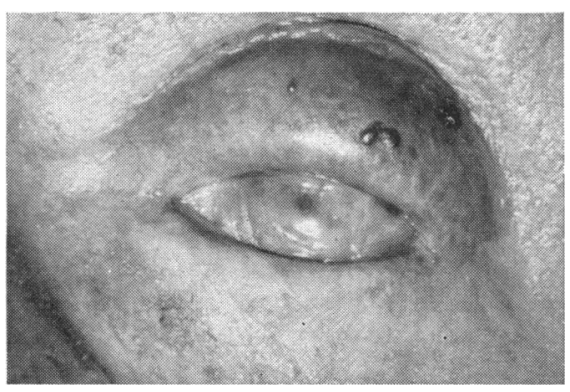

FIG. 5.--Final result.

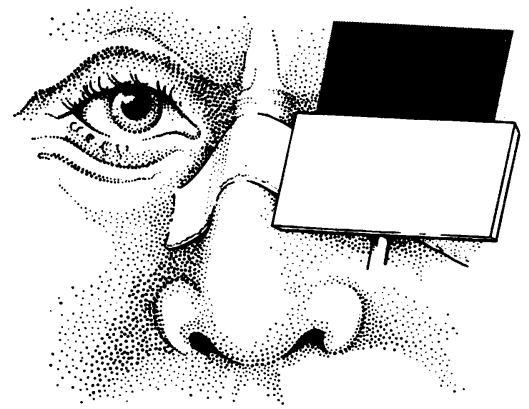

FIG. 4.-Diagram showing lead shield covering previously treated lower lid, corneal shield in place, and darker area delineating field of treatment.

Apart from these special techniques, treatment was uneventful, but subsequently the severe reaction caused adhesions to form in the fornices. The eye was scarred but comfortable. Fig. 5 shows the final result. 